\title{
Cross-sectional and prospective mediating effects of dietary intake on the relationship between sedentary behaviour and body mass index in adolescents
}

Elly A. Fletcher ${ }^{1 *}$ D, Karen E. Lamb ${ }^{1}$, Sarah A. McNaughton', Sarah P. Garnett ${ }^{2}$, David W. Dunstan 1,3,4,5,6,7,8, Louise A. Baur ${ }^{2}$ and Jo Salmon ${ }^{1}$

\begin{abstract}
Background: Cross-sectional evidence suggests TV viewing, but not objectively-measured sedentary time or bouts of sedentary time, is consistently associated with body mass index (BMI) in adolescents. However, it is unclear whether dietary intake is a potential mediator of these relationships. The aim of this study was to explore the cross-sectional and prospective mediating effects of dietary intake on the association of sedentary behaviour with BMI z-score (zBMI) in a cohort of Australian adolescents.

Methods: Cross-sectional and prospective analyses were conducted in adolescents aged 12-15 years participating in the 2002/03 (baseline) and 2004/05 (follow-up) Nepean Growing Up Study. The independent variables were television (TV) viewing, an objective measure of total sedentary time and average sedentary bout duration, and the outcome variable zBMI. Using the Sobel-Goodman method with bootstrapping, mediation analyses were conducted examining three dietary components (discretionary foods, sugarsweetened beverages [SSB] and takeaway foods) as mediators of associations between TV viewing and zBMI $(n=259)$ and between total sedentary time and average sedentary bout duration with zBMI $(n=140)$.
\end{abstract}

Results: No significant cross-sectional or prospective total or direct associations were observed for TV viewing, total sedentary time and average sedentary bout duration with zBMI. However, TV viewing was positively associated with consumption of takeaway foods cross-sectionally $(\beta=0.06 ; 95 \% \mathrm{Cl} 0.01$ to 0.12 ), prospectively at baseline ( $\beta=0.07 ; 95 \% \mathrm{Cl} 0.01$ to 0.12 ) and prospectively at follow-up ( $\beta=0.10 ; 95 \% \mathrm{Cl} 0.04,0.16$ ), and average sedentary bout duration was inversely associated with SSB consumption both cross-sectionally $(\beta=-0.36 ; 95 \% \mathrm{Cl}-0.69$ to -0.02$)$ and prospectively at baseline $(\beta=-0.36 ; 95 \% \mathrm{Cl}-0.70$ to -0.02$)$. No mediation effects were identified.

Conclusions: TV viewing, total sedentary time and bouts of sedentary time were not associated crosssectionally or prospectively with adolescents' zBMI, and three elements of dietary intake (e.g. intake of discretionary foods, SSB and takeaway foods) did not mediate this relationship. The role of dietary intake and sedentary behaviour in relation to adolescent health requires further clarification.

Keywords: Television viewing, Sedentary behaviour, Dietary intake, BMI, Adolescents

\footnotetext{
* Correspondence: elly.fletcher@deakin.edu.au

${ }^{1}$ Deakin University, Geelong, Australia, Institute for Physical Activity and

Nutrition (IPAN), School of Exercise and Nutrition Sciences, Geelong, Australia

Full list of author information is available at the end of the article
} 


\section{Background}

Adolescent obesity is a major public health concern. The combined rates of overweight and obesity among adolescents have increased over the last two decades worldwide [1]. In the United States, the proportion of obese adolescents has risen from $10.5 \%$ in $1988-1994$ to $20.6 \%$ in 2013-2014 [2]. Australia has experienced similar increases with almost one in three adolescents currently overweight or obese [3]. Given that obesity tracks from adolescence to adulthood [4], it is imperative to understand the lifestyle risk factors associated with adolescent obesity, particularly prospectively, in order to inform effective interventions.

Sedentary behaviour - defined as any waking behaviours characterised by low energy expenditure $(<1.5$ METS) while in a sitting or reclining posture - has emerged as a new research focus for obesity prevention [5]. High amounts of television (TV) viewing, a common leisure-time sedentary behaviour, during adolescence have both immediate and long-term health consequences, including a higher risk of obesity [6]. However, there are inconsistent associations between total time spent in sedentary time $[7,8]$, or time spent in periods, or 'bouts', of sedentary time [9], and indicators of adiposity (e.g. BMI, waist circumference) in adolescents.

One potential behavioural mechanism that could explain why TV viewing has more consistent associations with body mass index (BMI) when compared to total or bouts of sedentary time in adolescents, is an increase in energy-dense, nutrient poor foods and sugarsweetened beverages (SSB). For example, TV viewing has consistently been reported to be associated with a higher energy intake, and an increased consumption of discretionary foods, SSB, and fast food/takeaway foods in adolescents [10], whereas few studies have reported associations between objectively-measures of sedentary time with dietary intake $[11,12]$. In addition, no study to date has explored whether prolonged bouts of sedentary time are related to dietary intake among adolescents. The latter is important as studies with adults have shown that, independent of how much total sedentary time is accumulated, those with fewer interruptions in sedentary time (i.e., prolonged bouts) have poorer cardiometabolic health profiles [13].

A systematic review examining whether associations between sedentary behaviour and health outcomes in adolescents were independent of dietary intake found $\mathrm{TV}$ viewing, screen time and overall sedentary time were positively related to BMI, independent of dietary intake [14]. The systematic review also identified very few studies had specifically examined the mediating role of dietary intake in the TV viewing and BMI relation; with only two studies (out of the 21 studies identified) exploring this and reporting no mediation effects
$[15,16]$. However, a major limitation of both of these studies was their cross-sectional design which limits causal inference. Whereas, a longitudinal design would allow both the temporal order of associations to be examined and many other aspects of a mediation model to be explored.

Against this background, the primary aim of the study was to explore both the cross-sectional and prospective mediating effects of the consumption of discretionary foods, SSB and takeaway foods on the association between TV viewing and BMI z-score (zBMI) in a cohort of Australian adolescents, and to examine whether these findings differ when total sedentary time and sedentary bout duration are examined. The secondary aims of the study were to explore the individual associations between the sedentary behaviour variables (TV viewing, sedentary time and sedentary bout duration) and dietary intake variables (discretionary foods, SSB and takeaway foods), as well as their associations with zBMI. Based on the existing evidence, we hypothesised that consumption of discretionary foods, SSB and takeaway foods would partially mediate the cross-sectional and prospective association between TV viewing and zBMI, but would not mediate the crosssectional or prospective association between total sedentary time and sedentary bouts with zBMI.

\section{Methods}

Study design

In 2002/03 (baseline), 348 adolescents aged $12-13$ years participated in the Nepean Kids Growing-Up Study. The adolescents were originally part of a birth cohort study (the "Nepean Study") which involved 2314 infants born between 1989 and 1990 at the Nepean Hospital (western Sydney, Australia). Written consent was obtained from the parent or guardian and assent from the adolescent. The Ethics Committees of The Children's Hospital at Westmead and Wentworth Area Health Service gave ethical approval. Full details about the original study and eligibility criteria have been previously published [17]. Briefly, the study involved adolescents attending the clinic at Nepean Hospital, where they had their height and weight measured and completed a questionnaire on their demographics and physical activity levels, and a semi-quantitative food frequency questionnaire (FFQ). Afterwards, adolescents wore an accelerometer for 7 days during all waking hours. In 2004/05, the adolescents were recontacted and invited to participate in the follow-up study. In total, 63 adolescents were unable to be contacted or withdrew from the study, leaving 285 adolescents participating at both time points.

\section{Outcome variable (zBMI)}

Height and weight were measured at both time points by a trained research assistant and study dietitian at the 
clinic. Height was measured to the nearest $0.1 \mathrm{~cm}$ and weight was measured without shoes and in light clothing to the nearest $0.1 \mathrm{~kg}$ with electronic scales (Wedderburn, Summer Hill, NSW, Australia). Height and weight were used to calculate each participants' BMI and zBMI was determined using the age- and sex-specific CDC 2000 reference data [18]. Overweight and obesity was determined using the International Obesity Task Force (IOTF) criteria [19]. In all analyses, zBMI was treated as a continuous variable.

\section{Independent variables (sedentary behaviour)}

Adolescents completed a self-report questionnaire on their time spent watching TV (hours/day) on a usual school day (Monday to Friday) and a usual weekend day (Saturday and Sunday). The questionnaire has previously been shown to have good to excellent reliability (percentage agreement $=70 \%-99 \%)[20]$. To calculate average daily hours spent watching TV over a usual week, daily weekday TV hours was multiplied by five and daily weekend TV hours was multiplied by 2 , then summed together and divided by seven.

Sedentary time was measured objectively by an ActiGraph AM-7164 accelerometer (ActiGraph Inc., Florida). At both time points, adolescents were asked to wear the monitor on their right hip during all waking hours for 7 days, except when bathing, swimming and sleeping. Data were downloaded in 1-min epochs and non-wear time was defined as at least $20 \mathrm{~min}$ of zero counts. Sedentary time was defined as all wear-time minutes with an average activity count of $\leq 100$ counts per minute (cpm), and was standardised for wear time using the residual method [21]. Average sedentary bout duration was calculated by summing all uninterrupted minutes $\leq 100 \mathrm{cpm}$, and then taking the midpoint of all sedentary bouts that lie on the accumulation curve for each individual [22]. Analyses were limited to participants who had $\geq 8 \mathrm{~h}$ of wear time on $\geq 3$ week days and $\geq 7 \mathrm{~h}$ of wear time on $\geq 1$ weekend day [23].

\section{Mediating variables (dietary intake)}

Usual dietary intake was measured using a 56-item semi-quantitative FFQ, which was developed based on data from the 1995 Australian National Nutrition Survey [24]. Adolescents were asked to report how often they ate certain foods and beverages (either in times per week or per day) over the previous 7 days. The 8 -item frequency response scale was converted to times per week as follows: 1) "not consumed last week" $=0 ; 2$ ) "consumed once last week" $=0.143$; 3) "consumed $2-3$ times last week" (average number used) $=0.357$; 4) "consumed 4-6 times last week" (average number used) $=0.714 ; 5)$ "consumed once a day" $=1$; 6) "consumed 2 times a day" $=2$; 7) "consumed 3 times a day" = 3 ; 8) "consumed 4-6 times a day" (average number used) $=5$. For the analyses, a combination of food and beverage items were summed together to create three dietary mediators at both time points: 1) frequency of consuming discretionary foods, which included any savoury or plain biscuits, sweet pastries, cakes, doughnuts, chocolate, confectionary, and potato chips; 2) frequency of consuming SSB, which included non-diet soft drink, non-diet cordial and fruit juice and not sweetened milk drinks or energy drinks; and 3) frequency of consuming takeaway foods, which included savoury pastries (e.g. meat pies and sausage rolls), hamburgers, pizza, hot chips and spring rolls/dim sims. The FFQ was tested for reproducibility and overall showed fair to excellent reliability for sweet snacks $(\mathrm{ICC}=0.61$ ), savoury snacks $(\mathrm{ICC}=0.63)$, SSBs $(\mathrm{ICC}=0.77)$, and fast food $(\mathrm{ICC}=0.44)$. Frequency of consuming discretionary foods and frequency of consuming SSB were multiplied by 7 to convert to times per day. Participants with missing data for any of the dietary items listed were excluded $(n=2)$. In all analyses, the dietary mediators were treated as continuous variables.

\section{Covariates}

The covariates considered for the analyses included age at baseline, sex, maternal education (an indicator for family socioeconomic status), pubertal status and accelerometry-measured moderate-to-vigorous physical activity (MVPA) collected at baseline. Maternal education was collapsed into three categories: "low" (some secondary education or less); "medium" (completing secondary school, an apprenticeship or technical certificate); and "high" (university or tertiary qualification). Pubertal status was self-assessed using the 'Tanner Stages' of breast development and commencement of menses (girls) and pubic hair and genitalia (boys) [25]. For analyses, participants were categorised as early puberty, mid-pubertal, late-pubertal and post-pubertal. Accelerometry-measured MVPA at baseline was calculated based on the Freedson accelerometer age-cut points [26] and considered as a covariate for the analyses involving sedentary time and sedentary bouts with zBMI.

\section{Statistical analyses}

As shown in Fig. 1, to be included in the analyses involving TV viewing and zBMI, participants were required to have complete data for age, sex, maternal education, pubertal status, dietary intake and TV viewing at baseline, and complete data for zBMI at baseline and follow-up $(n=259)$. To examine the association of total and bouts of sedentary time, dietary intake and zBMI, separate analyses were undertaken from a subsample of participants who met the previous inclusion criteria as well as 


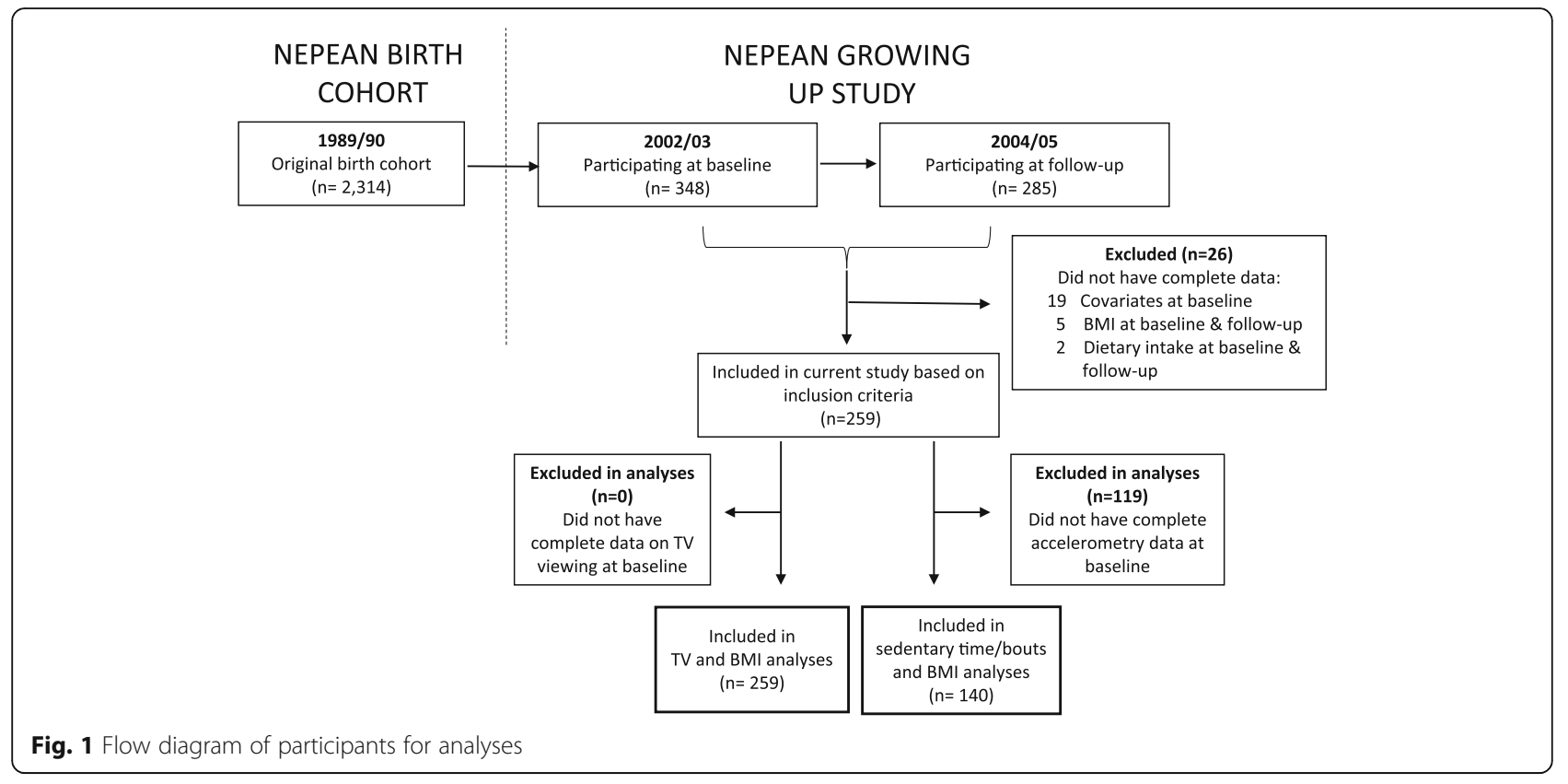

meeting the accelerometry inclusion criteria at baseline $(n=140)$. Prior to the main analyses, all variables were checked for normality. Discretionary foods, SSB and takeaway food intake at baseline were not normally distributed and were log-transformed.

Analyses were conducted using Stata/SE v14.0 (StataCorp LP, College Station, Texas, 2015). Figure 2 illustrates two theoretical models of the cross-sectional and prospective mediation pathways examined [27]. For all mediation analyses, the Sobel-Goodman mediation method with bootstrapping with 5000 replications was used to estimate standard errors and 95\% confidence intervals [28]. For the cross-sectional analyses, only baseline variables were used to test the following associations: 1) association between the independent variable and the mediator (a-coefficient pathway); 2) association between the mediator and the outcome variable, adjusting for the independent variable (b-coefficient pathway); 3) total association between the independent variable and outcome variable (c-coefficient pathway); 4) direct association between the independent variable and outcome variable, accounting for each mediator (c'-coefficient pathway); and 5) indirect association (e.g. mediating effect) of the mediator on the independent variable and outcome variable. For the prospective analyses, similar pathways were tested. However, dietary intake at baseline and dietary intake at follow-up were examined separately as potential mediators in the associations between the independent variable at baseline and the outcome variable at follow-up. All analyses were adjusted for age, sex, pubertal status and maternal education, with objectively-measured MVPA adjusted for in the analyses involving total and bouts of sedentary time

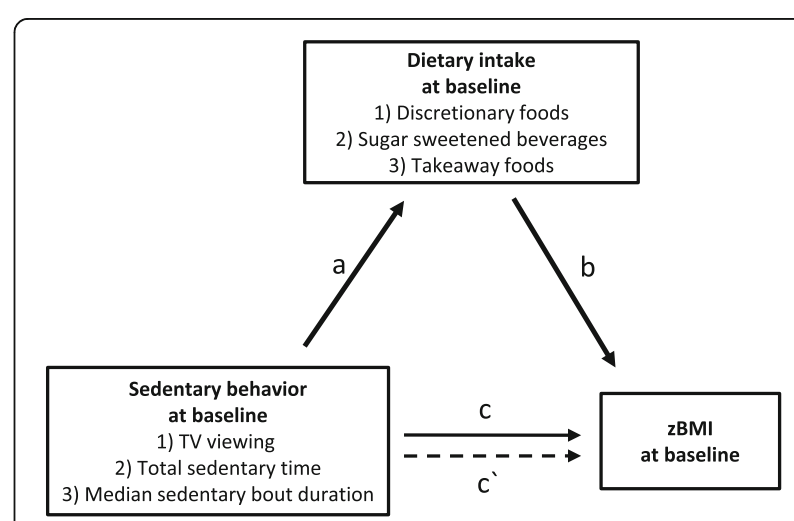

Model 1: Cross-sectional mediation pathways

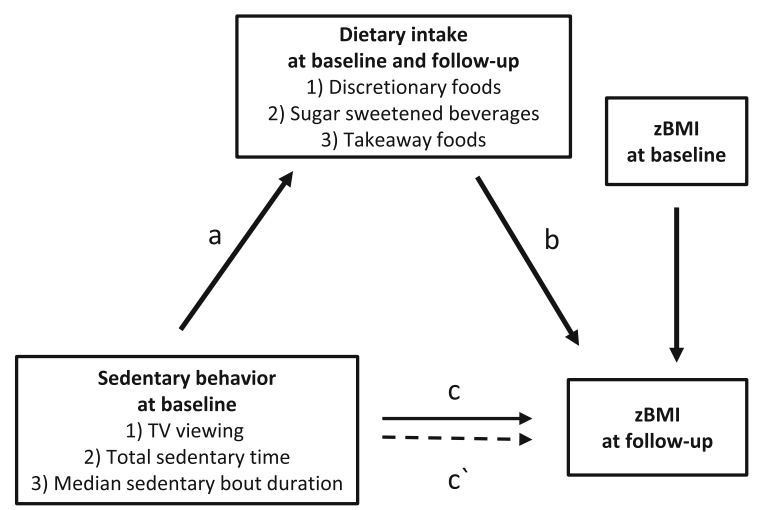

Model 2: Prospective mediation pathways

Fig. 2 Theoretical diagram of the cross-sectional (Model 1) and prospective (Model 2) mediation pathway 
with zBMI. The prospective analyses were additionally adjusted for zBMI at baseline. The significance level was set at $p<0.05$ for all statistical tests.

\section{Results}

Overall, 259 and 140 adolescents were included in the $\mathrm{TV}$ viewing and zBMI, and the sedentary time and zBMI analytic samples, respectively (Table 1 ). Those excluded in the TV viewing and zBMI analyses had mothers with a lower maternal education and those excluded in the

Table 1 Baseline characteristics of participants included in analyses

\begin{tabular}{|c|c|c|}
\hline Variables & $\begin{array}{l}\text { TV and } z B M l \\
(n=259)\end{array}$ & $\begin{array}{l}\text { Sedentary time } \\
\text { and zBMI }(n=140)\end{array}$ \\
\hline Age, years & $12.9(12.9,13.0)$ & $12.9(12.9,13.0)$ \\
\hline \multicolumn{3}{|l|}{ Sex, \% } \\
\hline Male & $47.5(41.4,53.6)$ & $50.0(41.7,58.3)$ \\
\hline Female & $52.5(46.4,58.4)$ & $50.0(41.7,58.3)$ \\
\hline \multicolumn{3}{|l|}{ Maternal education, $\%$} \\
\hline Low & $6.9(4.4,10.8)$ & $5.0(2.3,10.2)$ \\
\hline Medium & $65.3(59.2,70.8)$ & $62.8(54.4,70.5)$ \\
\hline High & $27.8(22.7,33.6)$ & $32.1(24.8,40.4)$ \\
\hline \multicolumn{3}{|l|}{ Pubertal status, $\%$} \\
\hline Early puberty & $19.3(8.0,4.6)$ & $14.3(35.1,56.2)$ \\
\hline Mid-pubertal & $35.5(30.0,41.6)$ & $41.4(33.5,50.0)$ \\
\hline Late-pubertal & $35.5(30.0,41.6)$ & $35.0(27.5,43.4)$ \\
\hline Post-pubertal & $27.0(21.9,32.8)$ & $22.1(16.0,30.0)$ \\
\hline \multicolumn{3}{|l|}{ BMI } \\
\hline $\mathrm{kg} / \mathrm{m}^{2}$ & $20.7(20.2,21.3)$ & $20.3(19.7,21.0)$ \\
\hline z-score & $0.4(0.3,0.5)$ & $0.3(0.1,0.5)$ \\
\hline Overweight, \% & $18.9(14.6,24.2)$ & $13.6(8.8,20.4)$ \\
\hline Obese, \% & $9.2(6.3,13.5)$ & $9.2(5.4,15.4)$ \\
\hline \multicolumn{3}{|l|}{ Sedentary behaviour } \\
\hline TV viewing (hours/day) & $3.2(3.0,3.4)$ & \\
\hline Total sedentary time (hours/day) & & $6.1(6.0,6.2)$ \\
\hline $\begin{array}{l}\text { Average sedentary bout } \\
\text { (minutes/day) }\end{array}$ & & $6.6(6.5,6.6)$ \\
\hline MVPA (minutes/day) & & $48.2(35.0,61.4)$ \\
\hline \multicolumn{3}{|l|}{ Dietary intake } \\
\hline Discretionary foods (freq/day) & $2.4(2.2,2.6)$ & $2.4(2.1,2.7)$ \\
\hline $\begin{array}{l}\text { Sugar-sweetened beverages } \\
\text { (freq/day) }\end{array}$ & $2.1(1.8,2.3)$ & $1.9(1.6,2.2)$ \\
\hline Takeaway food (freq/week) & $3.4(3.1,3.6)$ & $3.2(2.8,3.6)$ \\
\hline
\end{tabular}

$B M I$ body mass index, zBMI BMI z-score, TV television, MVPA moderate-tovigorous physical activity; freq: frequency

Footnote: Maternal education: low = some secondary education or less; medium $=$ completing secondary school, an apprenticeship or technical certificate; high = university or tertiary qualification. Pubertal status self-assessed using the 'Tanner Stages' [25]. BMI z-score determined using the age- and sex-specific CDC 2000 reference data [18]. Overweight and obesity determined using the International Obesity Task Force (IOTF) criteria [19] sedentary time and zBMI analyses were older, had a lower maternal education and had a higher proportion of overweight participants (Additional file 1: Tables S1 and $\mathrm{S} 2$ in the online supplement file).

\section{Associations between TV viewing, dietary intake and zBMI Cross-sectionally}

There was no evidence of total or direct cross-sectional associations of TV viewing with zBMI (Table 2). A small, positive association was observed between hours spent watching TV per day and frequency of consuming takeaway foods each week (a-coefficient pathway); with each additional hour of TV viewing, adolescents consumed an additional 0.06 serves (95\% CI 0.01 to $0.12 ; p<0.05)$ of takeaway foods each week. An inverse association was also observed for consuming discretionary foods each day and zBMI (b-coefficient pathway); with each additional serving of discretionary foods consumed each day, zBMI was lower by -0.39 units $(95 \%$ CI -0.65 to $-0.13 ; p<0.01)$. None of the dietary variables were significant mediators of the cross-sectional association of TV viewing with zBMI.

\section{Prospectively}

There was no evidence of total or direct prospective associations between TV viewing at baseline and zBMI at follow-up. Similar to the cross-sectional associations, a positive prospective association was observed between hours spent watching TV per day and frequency of consuming takeaway foods each week at baseline and at follow-up. For example, for each additional hour of TV viewing, adolescents consumed an additional 0.07 (95\% CI 0.01 to $0.12 ; p<0.05$ ) serves of take-away foods each week at baseline, and an additional 0.10 (95\% CI 0.04 to 0.16 ; $p<0.05)$ serves of take-away foods each week at follow-up. However, no significant association remained for any of the dietary variables consumed at baseline and at follow-up with zBMI at follow-up (b-coefficient pathway). None of the dietary variables were significant mediators of the prospective association between TV viewing and zBMI.

\section{Associations between total sedentary time, dietary intake and zBMI \\ Cross-sectionally}

There was no evidence of total or direct cross-sectional associations for total sedentary time (hours/day) and zBMI, or between total sedentary time and any of the dietary mediators (a-coefficient pathway) (Table 3). An inverse association was observed between frequency of consuming discretionary foods per day and zBMI; with each additional discretionary food consumed each week, zBMI was lower by -0.42 units $(95 \%$ CI -0.77 to $-0.07 ; p<0.05)$ (b-coefficient). There were no significant mediating effects for any of the dietary variables in the cross-sectional association of total sedentary time with zBMI. 
Table 2 Cross-sectional ${ }^{a}$ and prospective ${ }^{b}$ associations of dietary intake (mediator), TV viewing (independent) and zBMI (outcome) $(n=259)$

\begin{tabular}{|c|c|c|c|c|c|}
\hline \multirow{2}{*}{$\begin{array}{l}\text { Outcome: zBMI } \\
\text { Independent: TV viewing }\end{array}$} & \multirow{2}{*}{$\begin{array}{l}\text { c'-coefficient } \\
\text { (direct) } \beta(95 \% \text { Cl) }\end{array}$} & \multirow{2}{*}{$\begin{array}{l}\text { a-coefficient } \beta \\
(95 \% \mathrm{Cl})\end{array}$} & \multirow{2}{*}{$\begin{array}{l}\text { b-coefficient } \beta \text { (95\% } \\
\text { Cl) }\end{array}$} & \multicolumn{2}{|c|}{$\mathrm{ab} /$ indirect $(\text { mediated/indirect) })^{c}$} \\
\hline & & & & Uncorrected $\beta$ (95\% Cl) & Bias-corrected $\beta$ (95\% Cl) \\
\hline \multicolumn{6}{|l|}{ Cross-sectional mediators } \\
\hline Discretionary food (freq/day) & $0.05(-0.02,0.13)$ & $0.01(-0.03,0.05)$ & $-0.39(-0.65,-0.13)^{* *}$ & $-0.04(-0.18,0.10)$ & $-0.04(-0.23,0.12)$ \\
\hline SSB (freq/day) & $0.05(-0.03,0.13)$ & $0.02(-0.03,0.07)$ & $0.03(-0.15,0.22)$ & $0.01(-0.04,0.05)$ & $0.01(-0.07,0.09)$ \\
\hline Takeaway food (freq/week) & $0.05(-0.02,0.13)$ & $0.06(0.01,0.12)^{*}$ & $-0.07(-0.24,0.09)$ & $-0.05(-0.16,0.07)$ & $-0.05(-0.22,0.06)$ \\
\hline \multicolumn{6}{|l|}{ Prospective mediators } \\
\hline \multicolumn{6}{|l|}{ At baseline } \\
\hline Discretionary foods (freq/day) & $-0.01(-0.04,0.02)$ & $0.01(-0.02,0.05)$ & $0.02(-0.09,0.13)$ & $0.01(-0.01,0.02)$ & $0.01(-0.02,0.04)$ \\
\hline SSB (freq/day) & $-0.01(-0.04,0.02)$ & $0.02(-0.03,0.07)$ & $0.03(-0.04,0.11)$ & $0.01(-0.02,0.03)$ & $0.01(-0.02,0.04)$ \\
\hline Takeaway food (freq/week) & $-0.01(-0.04,0.02)$ & $0.07(0.01,0.12)^{*}$ & $-0.01(-0.07,0.06)$ & $-0.01(-0.04,0.05)$ & $-0.01(-0.06,0.05)$ \\
\hline \multicolumn{6}{|l|}{ At follow-up } \\
\hline Discretionary foods (freq/day) & $-0.01(-0.04,0.02)$ & $0.02(-0.02,0.07)$ & $-0.04(-0.13,0.06)$ & $-0.01(-0.04,0.02)$ & $-0.01(-0.06,0.02)$ \\
\hline SSB (freq/day) & $-0.01(-0.04,0.02)$ & $-0.01(-0.05,0.04)$ & $0.03(-0.06,0.11)$ & $-0.001(-0.01,0.01)$ & $-0.001(-0.03,0.02)$ \\
\hline Takeaway food (freq/week) & $-0.01(-0.04,0.02)$ & $0.10(0.04,0.16)^{*}$ & $0.02(-0.05,0.08)$ & $0.02(-0.05,0.08)$ & $0.02(-0.04,0.09)$ \\
\hline
\end{tabular}

${ }^{a}$ Cross-sectional total effects (c-pathway) of TV viewing and zBMI $\beta=0.05$ ( $95 \% \mathrm{Cl}-0.03$ to 0.13 ), adjusting for age, sex, mother's education and pubertal status. ${ }^{b}$ Prospective total effects (c-pathway) of TV viewing and zBMI $\beta=-0.01$ ( $95 \% \mathrm{Cl}-0.04$ to 0.02 ), adjusting for age, sex, mother's education, pubertal status and zBMI at baseline. 'Due to the small units of measure, the indirect effects have been multiplied by 10

Significant ${ }^{* *} p<0.01,{ }^{*} p<0.05$. TV: television; SSB: sugar-sweetened beverages; zBMI: body mass index z-score; freq: frequency

\section{Prospectively}

Total sedentary time at baseline was not significantly associated with zBMI at follow-up, accounting for mediation by dietary intake at baseline and at follow-up. No significant associations were also observed for any of the total, direct and indirect pathways, nor with the acoefficient and b-coefficient pathways.

\section{Associations between average sedentary bout duration, dietary intake and zBMI Cross-sectionally}

No significant total or direct cross-sectional associations were observed for average sedentary bout duration (minutes/day) with zBMI (Table 4). Average sedentary bout duration was inversely related to frequency of

Table 3 Cross-sectional $^{\mathrm{a}}$ and prospective ${ }^{\mathrm{b}}$ associations of dietary intake (mediator), sedentary time (independent) and zBMl (outcome) $(n=140)$

\begin{tabular}{|c|c|c|c|c|c|}
\hline \multirow{2}{*}{$\begin{array}{l}\text { Outcome: zBMI } \\
\text { Independent: total sedentary time }\end{array}$} & \multirow{2}{*}{$\begin{array}{l}\text { c'-coefficient (direct) } \\
\beta(95 \% \mathrm{Cl})\end{array}$} & \multirow{2}{*}{$\begin{array}{l}\text { a-coefficient } \\
\beta(95 \% \mathrm{Cl})\end{array}$} & \multirow{2}{*}{$\begin{array}{l}\text { b-coefficient } \\
\beta(95 \% \mathrm{Cl})\end{array}$} & \multicolumn{2}{|c|}{$\mathrm{ab} /$ indirect $\left(\right.$ mediated/indirect) ${ }^{c}$} \\
\hline & & & & Uncorrected $\beta$ (95\% CI) & Bias-corrected $\beta$ (SE) \\
\hline \multicolumn{6}{|l|}{ Cross-sectional mediators } \\
\hline Discretionary food (freq/day) & $0.27(-0.03,0.58)$ & $-0.01(-0.15,0.14)$ & $-0.42(-0.77,-0.07)^{*}$ & $0.01(-0.61,0.62)$ & $0.01(-0.62,0.69)$ \\
\hline SSB (freq/day) & $0.27(-0.05,0.58)$ & $-0.16(-0.38,0.06)$ & $-0.04(-0.29,0.20)$ & $0.07(-0.33,0.46)$ & $0.07(-0.31,0.61)$ \\
\hline Takeaway food (freq/week) & $0.27(-0.05,0.58)$ & $-0.06(-0.30,0.18)$ & $-0.04(-0.26,0.18)$ & $0.02(-0.13,0.18)$ & $0.02(-0.36,0.35)$ \\
\hline \multicolumn{6}{|l|}{ Prospective mediators } \\
\hline \multicolumn{6}{|l|}{ At baseline } \\
\hline Discretionary food (freq/day) & $0.05(-0.07,0.17)$ & $0.03(-0.12,0.17)$ & $0.03(-0.10,0.17)$ & $0.01(-0.05,0.07)$ & $0.01(-0.08,0.15)$ \\
\hline SSB (freq/day) & $0.05(-0.06,0.17)$ & $-0.15(-0.37,0.07)$ & $0.02(-0.07,0.11)$ & $-0.03(-0.17,0.11)$ & $-0.03(-0.22,0.13)$ \\
\hline Takeaway food (freq/week) & $0.05(-0.07,0.17)$ & $-0.05(-0.30,0.19)$ & $-0.03(-0.12,0.05)$ & $0.02(-0.08,0.11)$ & $0.02(-0.12,0.16)$ \\
\hline \multicolumn{6}{|l|}{ At follow-up } \\
\hline Discretionary food (freq/day) & $0.05(-0.07,0.17)$ & $0.08(-0.09,0.24)$ & $0.01(-0.11,0.13)$ & $0.01(-0.05,0.10)$ & $0.01(-0.16,0.17)$ \\
\hline SSB (freq/day) & $0.05(-0.06,0.17)$ & $-0.04(-0.23,0.15)$ & $0.01(-0.10,0.12)$ & $-0.01(-0.05,0.04)$ & $-0.01(-0.11,0.09)$ \\
\hline Takeaway food (freq/week) & $0.05(-0.06,0.17)$ & $0.21(-0.57,0.47)$ & $-0.01(-0.08,0.07)$ & $-0.01(-0.16,0.15)$ & $-0.01(-0.19,0.17)$ \\
\hline
\end{tabular}

${ }^{a}$ Cross-sectional total effects (c-pathway) of total sedentary time and zBMI $\beta=0.27(95 \% \mathrm{Cl}-0.04$ to 0.58$)$, adjusting for age, sex, mother's education and pubertal status. ${ }^{b}$ Prospective total effects (c-pathway) of total sedentary time and zBMI $\beta=0.05(95 \% \mathrm{Cl}-0.06$ to 0.17$)$, adjusting for age, sex, mother's education, pubertal status and zBMI at baseline. 'Due to the small units of measure, the indirect effects have been multiplied by 10 . Significant ${ }^{*} p<0.05$. SSB: sugar-sweetened beverages; $z B M I$ : body mass index z-score; freq: frequency 
Table 4 Cross-sectional $^{a}$ and prospective ${ }^{b}$ associations of dietary intake (mediator), sedentary bouts (independent) and zBMI (outcome) $(n=140)$

\begin{tabular}{|c|c|c|c|c|c|}
\hline \multirow{2}{*}{$\begin{array}{l}\text { Outcome: zBMI } \\
\text { Independent: sedentary bouts }\end{array}$} & \multirow{2}{*}{$\begin{array}{l}c^{\prime} \text {-coefficient (direct) } \\
\beta(95 \% \mathrm{Cl})\end{array}$} & \multirow{2}{*}{$\begin{array}{l}\text { a-coefficient } \\
\beta(95 \% \mathrm{Cl})\end{array}$} & \multirow{2}{*}{$\begin{array}{l}\text { b-coefficient } \\
\beta(95 \% \mathrm{Cl})\end{array}$} & \multicolumn{2}{|c|}{$\mathrm{ab} /$ indirect $\left(\right.$ mediated/indirect) ${ }^{c}$} \\
\hline & & & & Uncorrected $\beta$ (95\% Cl) & Bias-corrected $\beta$ (SE) \\
\hline \multicolumn{6}{|l|}{ Cross-sectional mediators } \\
\hline Discretionary food (freq/day) & $0.12(-0.36,0.61)$ & $-0.08(-0.31,0.15)$ & $-0.42(-0.77,-0.06)^{*}$ & $0.32(-0.67,1.32)$ & $0.32(-0.55,1.37)$ \\
\hline SSB (freq/day) & $0.14(-0.36,0.64)$ & $-0.36(-0.69,-0.02)^{*}$ & $-0.06(-0.30,0.19)$ & $0.20(-0.71,1.11)$ & $0.20(-0.68,0.12)$ \\
\hline Takeaway food (freq/week) & $0.16(-0.33,0.65)$ & $0.03(-0.34,0.41)$ & $-0.05(-0.28,0.18)$ & $-0.02(-0.21,0.18)$ & $-0.02(-0.62,0.34)$ \\
\hline \multicolumn{6}{|l|}{ Prospective mediators } \\
\hline \multicolumn{6}{|l|}{ At baseline } \\
\hline Discretionary food (freq/day) & $0.08(-0.10,0.26)$ & $-0.06(-0.29,0.17)$ & $0.04(-0.10,0.17)$ & $-0.02(-0.14,0.10)$ & $-0.02(-0.24,0.14)$ \\
\hline SSB (freq/day) & $0.09(-0.09,0.27)$ & $-0.36(-0.70,-0.02)^{*}$ & $0.02(-0.07,0.11)$ & $-0.09(-0.42,0.24)$ & $-0.09(-0.52,0.24)$ \\
\hline Takeaway food (freq/week) & $0.08(-0.10,0.26)$ & $0.04(-0.34,0.41)$ & $-0.04(-0.12,0.04)$ & $-0.01(-0.15,0.13)$ & $-0.01(-0.27,0.16)$ \\
\hline \multicolumn{6}{|l|}{ At follow-up } \\
\hline Discretionary food (freq/day) & $0.08(-0.10,0.26)$ & $-0.08(-0.34,0.17)$ & $0.02(-0.10,0.14)$ & $-0.02(-0.12,0.09)$ & $-0.02(-0.28,0.16)$ \\
\hline SSB (freq/day) & $0.08(-0.10,0.26)$ & $0.11(-0.18,0.40)$ & $0.01(-0.010,0.11)$ & $0.01(-0.11,0.12)$ & $0.01(-0.19,0.20)$ \\
\hline Takeaway food (freq/week) & $0.08(-0.10,0.26)$ & $0.06(-0.35,0.48)$ & $-0.01(-0.08,0.07)$ & $-0.01(-0.05,0.04)$ & $-0.01(0.18,0.17)$ \\
\hline
\end{tabular}

${ }^{a}$ Cross-sectional total effects (c-pathway) of average sedentary bout and zBMI $\beta=0.16(95 \% \mathrm{Cl}-0.33$ to 0.65$)$, adjusting for age, sex, mother's education and pubertal status. ${ }^{b}$ Prospective total effects (c-pathway) of average sedentary bout and $\mathrm{zBMI} \beta=0.08$ ( $95 \% \mathrm{Cl}-0.10$ to 0.26$)$, adjusting for age, sex, mother's education, pubertal status and zBMI at baseline. ${ }^{c}$ Due to the small units of measure, the indirect effects have been multiplied by 10 . Significant ${ }^{*} p<0.05$. SSB: sugar-sweetened beverages; zBMI: body mass index z-score; freq: frequency

consuming SSB each day (a-coefficient pathway); with each additional minute spent in a sedentary bout, the frequency of consuming SSB was lower by nearly half a serve each day $(\beta=-0.36,95 \%$ CI -0.69 to -0.02 ; $p<0.05)$. An inverse association was also observed for frequency of consuming discretionary foods each week and zBMI (b-coefficient pathway); with each additional discretionary food consumed each week, zBMI was lower by -0.42 units $(95 \%$ CI -0.77 to $-0.06 ; p<0.05)$. None of the dietary variables significantly mediated cross-sectional associations of sedentary bout duration with zBMI.

\section{Prospectively}

When examining the prospective associations between average sedentary bout duration at baseline and zBMI at follow-up, no significant total or direct associations were observed. However, a significant inverse association remained for average sedentary bout duration with $f$ requency of consuming SSB at baseline $(\beta=-0.36$; $95 \%$ CI -0.70 to $-0.02 ; p<0.05$ ), but not at follow-up for the a-coefficient pathways. No significant associations were observed for the b-coefficient pathways or mediating effects.

\section{Discussion}

This study found no evidence of direct or indirect associations for TV viewing, total sedentary time and average sedentary bout duration with adolescents' zBMI, either cross-sectionally or prospectively. Although some of the dietary variables were independently associated with TV viewing, average sedentary bout duration and zBMI, none of the dietary variables significantly mediated the relationships between the sedentary variables and zBMI cross-sectionally or prospectively.

The null finding for the association of TV viewing with zBMI in the current study is in contrast to previous studies in youth that have consistently shown significant and positive associations both cross-sectionally [6] and prospectively $[29,30]$. The differences in findings could be attributed to the homogeneity of the current sample being examined, with higher than average number of hours spent watching TV [31] and a lower zBMI compared to the population average [32]. The null association for total sedentary time and average sedentary bout duration with zBMI is supported by some previous studies [8, 33-35], but not others [7]. Research examining accelerometer-measured sedentary time with health indictors among children and youth appears to be mixed; it is unclear whether an association exists in only some populations or if there are inconsistencies in measuring sedentary time and/or the analytical approaches undertaken.

The positive association observed for TV viewing with the consumption of takeaway foods, both cross-sectionally and prospectively is consistent with previous research [36, 37]. This link could be partially explained by the large extent of TV advertising of foods high in fat and energy during peak times when children and adolescents are likely to be watching TV [38]. In contrast, no evidence of an association was observed for total sedentary time with any of the dietary 
variables. The null finding is consistent with previous literature in youth $[11,12]$ where, compared to TV viewing, fewer significant associations are observed for total sedentary time with elements of a less healthy diet. The null finding could be due to the measure used to capture total sedentary time. For example, accelerometers measure all time spent being sedentary, and thus may capture times where adolescents may not be eating/drinking (e.g. sitting in school, sitting in the car). In addition, due to accelerometers being unable to determine posture (e.g. standing still versus sitting), time spent standing may have been included in total sedentary time and thus may diminish the opportunity to engage in an eating occasion. Unexpectedly, the study found higher sedentary bout duration was inversely associated with a lower consumption of SSB, both crosssectionally and prospectively. One possible reason for this could be due to the adolescents not breaking up their sedentary time in order to retrieve a SSB from another room (e.g. the kitchen, school canteen). However, given the current study is one of the first to examine individual associations between sedentary bout duration and dietary intake, further research is urgently needed in this area.

In contrast, the cross-sectional, inverse association found for discretionary foods with zBMI, and the null finding for SSB and takeaway foods is in contrast with previous studies that have found positive associations for unhealthy dietary intake with BMI $[39,40]$. Our findings could be a consequence of under-reporting, where overweight or obese children and adolescents have been found to under-report their energy intake by $20-40 \%$ [41]. Alternatively, it is possible that some participants in the current study with a higher zBMI may have changed their behaviour by decreasing their discretionary food intake over time as a strategy to manage their weight.

Only one other study has explored the prospective associations between TV viewing, dietary intake and BMI; however, that study examined a younger population of pre-school children aged 0-5 years over a 2-year period [42]. In contrast to the current study, Fuller-Tyszkiewicz et al. reported a significant positive association between TV viewing and BMI that was bi-directional, with those children characterised with high amounts of TV viewing having higher BMI, and children with higher BMI watching a greater amount of TV. In addition, that study reported the prospective associations between TV viewing and BMI among 4 year olds were mediated by discretionary foods and soft drink consumption [42]. The differences in findings between the current study and previous study could be contributed to the different study populations and dietary mediators being examined, and that the previous study only examined TV viewing and BMI. Thus, further prospective studies are needed to explore whether dietary intake mediates the relationship between various sedentary behaviours (both subjective and objectively measured) and health indicators (e.g. BMI, metabolic syndrome).

To our knowledge, the current study is the first to examine both the cross-sectional and prospective mediating effects of dietary intake on the association between sedentary behaviour and zBMI in an adolescent population, and the first to examine this using objective measures of sedentary time. Other strengths include adjustment for a variety of confounders, including maternal education and adolescent pubertal status, and examining the dietary mediators (discretionary foods, SSB, takeaway foods) separately in all models. Limitations of the study include participants self-reporting their dietary intake using an FFQ and hours spent watching TV, and the low sample size for mediation analyses. In addition, the semi-quantitative FFQ used in the current study was limited with the number of healthy food items included (e.g. fruit and vegetables) and did not have information on portion sizes. There were also differences in those that were included and excluded in the analysis of this study and thus may limit the representative of the findings. Further, the data presented in the current study was collected more than a decade ago and thus the behaviours reported in the study may not reflect the contemporary sedentary and dietary behaviours adolescents are engaging in today.

\section{Conclusion}

In conclusion, despite identifying some significant associations between TV viewing and average sedentary bout duration with frequency of consuming takeaway foods and SSB, and between frequency of consuming discretionary foods and zBMI, no significant associations were observed for any of the sedentary behaviour variables with zBMI, either cross-sectionally or prospectively. In addition, none of the dietary variables were found to be significant mediators of the associations between sedentary behaviour and zBMI. Given the unacceptably high levels of adolescent overweight and obesity, further studies are warranted to elucidate the complex relationships between TV viewing, sedentary time, dietary intake and health indicators.

\section{Additional file}

Additional file 1: Supplement file. Comparison of baseline characteristics of participants included in the TV viewing and zBMI analyses compared to those excluded in the analyses. (PDF 29 kb)

\section{Abbreviations}

BMI: Body mass index; Cpm: Counts per minute; FFQ: Food frequency questionnaire; MVPA: Moderate-to-vigorous physical activity; SSB: Sugarsweetened beverages; TV: Television; zBMI: Body mass index z-score 


\section{Acknowledgements}

The authors would like to thank Dr. Vanessa Shrewsbury for collecting the data and her work on the project. We would like to thank all the families that generously donated their time to participate in this study.

\section{Funding}

The Nepean longitudinal study was funded by The Children's Hospital at Westmead Grant Research Scheme, a National Health and Medical Research Council Project Grant \#206501 and Meat and Livestock Australia. SAM is funded by an NHMRC Career Development Fellowship Level 2, ID1104636 and was previously funded by an ARC Future Fellowship (2011-2015, FT100100581). DWD is funded by NHMRC Senior Research Fellowship (APP1078360). JS is funded by a NHMRC Principal Research Fellowship (APP1026216). This work was also partially funded by an OIS grant from the Victorian State Government and a National Health \& Medical Research Council, Centre of Research Excellence grant (APP1057608).

\section{Availability of data and materials}

The dataset supporting the conclusions of this article has not been approved to be made publicly available by the ethics committees. To require information about the content and analyses of the dataset, contact Sarah Garnett: sarah.garnett@health.nsw.gov.au

\section{Authors' contributions}

$J S, L B$, and SG were involved in the conception and design of the study. $\mathrm{EF}$ carried out the data analysis with statistical guidance from $\mathrm{KL}$. EF led the writing of the article, with contributions from authors KL, SM, JS, LB, SG, and DD. All authors read and approved the final manuscript.

\section{Ethics approval and consent to participate}

Ethical approval was provided by the Ethics Committees of The Children's Hospital at Westmead (Project No: 2001/095) and Wentworth Area Health Service (Registration No 2001/065). Written consent was obtained from the adolescent's parent or guardian and each adolescent signed a study agreement form.

\section{Consent for publication}

Not applicable.

\section{Competing interests}

The authors declare that they have no competing interests.

\section{Publisher's Note}

Springer Nature remains neutral with regard to jurisdictional claims in published maps and institutional affiliations.

\section{Author details}

'Deakin University, Geelong, Australia, Institute for Physical Activity and Nutrition (IPAN), School of Exercise and Nutrition Sciences, Geelong, Australia. ${ }^{2}$ The Children's Hospital at Westmead Clinical School, University of Sydney, Westmead, Australia. ${ }^{3}$ Baker IDI Heart and Diabetes Institute, Melbourne, VIC, Australia. ${ }^{4}$ Department of Medicine, Monash University, Melbourne, Australia. ${ }^{5}$ The University of Queensland, School of Public Health, Brisbane, Australia. ${ }^{6}$ Department of Epidemiology and Preventive Medicine, Monash University, Melbourne, Australia. ' School of Sport Science, Exercise and Health, The University of Western Australia, Perth, Australia. ${ }^{8}$ Mary MacKillop Institute for Health Research, Australian Catholic University, Melbourne, Australia.

Received: 29 January 2017 Accepted: 18 September 2017

\section{Published online: 29 September 2017}

\section{References}

1. Wang Y, Lobstein T. Worldwide trends in childhood overweight and obesity. Int J Pediatr Obes. 2006;1(1):11-25.

2. Ogden CL, Carroll MD, Lawman HG, et al. Trends in obesity prevalence among children and adolescents in the united states, 1988-1994 through 2013-2014. JAMA. 2016;315(21):2292-9.

3. Australian Bureau of Statistics. Profiles of Health, Australia, 2011-13. 2013. http://www.abs.gov.au/.
4. Singh AS, Mulder C, Twisk JW, van Mechelen W, Chinapaw MJ. Tracking of childhood overweight into adulthood: a systematic review of the literature. Obes Rev. 2008;9(5):474-88.

5. Sedentary Behaviour Research Network. Letter to the Editor: standardized use of the terms "sedentary" and "sedentary behaviours". Appl Physiol Nutr Metab. 2012;37(3):540-2.

6. Tremblay MS, LeBlanc AG, Kho ME, Saunders TJ, Larouche R, Colley RC, Goldfield G, Connor GS. Systematic review of sedentary behaviour and health indicators in school-aged children and youth. Int J Behav Nutr Phys Act. 2011;8(98):98.

7. Martínez-Gómez D, Eisenmann JC, Gómez-Martínez S, Veses A, Marcos A, Veiga OL. Sedentary behavior, adiposity, and cardiovascular risk factors in adolescents: the AFINOS study. Rev Esp Cardiol. 2010;63(3):277-85.

8. Chaput JP, Lambert M, Mathieu ME, Tremblay MS, O' Loughlin J, Tremblay A. Physical activity vs. sedentary time: independent associations with adiposity in children. Pediatr Obes. 2012;7(3):251-8.

9. Colley R, Garriguet D, Janssen I, Wong S, Saunders T, Carson V, Tremblay M. The association between accelerometer-measured patterns of sedentary time and health risk in children and youth: results from the Canadian Health Measures Survey. BMC Public Health. 2013;13(1):1-9.

10. Pearson N, Biddle S. Sedentary behavior and dietary intake in children, adolescents, and adults: a systematic review. Am J Prev Med. 2011;41(2):178-88.

11. Vissers PAJ, Jones AP, van Sluijs EMF, Jennings A, Welch A, Cassidy A, Griffin SJ. Association between diet and physical activity and sedentary behaviours in 9-10-year-old British White children. Public Health. 2013;127(3):231-40.

12. Borghese MM, Tremblay MS, Leduc $G$, Boyer $C$, Bélanger $P$, LeBlanc AG, Francis C, Chaput J-P. Independent and combined associations of total sedentary time and television viewing time with food intake patterns of 911 year-old Canadian children. Appl Physiol Nutr Metab. 2014;39(8):937-43.

13. Healy GN, Dunstan DW, Salmon J, Cerin E, Shaw JE, Zimmet PZ, Owen N. Breaks in sedentary time: beneficial associations with metabolic risk. Diabetes Care. 2008;31(4):661-6.

14. Fletcher E, Leech R, McNaughton SA, Dunstan DW, Lacy KE, Salmon J. Is the relationship between sedentary behaviour and cardiometabolic health in adolescents independent of dietary intake? A systematic review. Obes Rev. 2015:16(9):795-805.

15. Carson V, Janssen I. The mediating effects of dietary habits on the relationship between television viewing and body mass index among youth. Pediatr Obes. 2012;7(5):391-8.

16. Fletcher EA, McNaughton SA, Lacy KE, Dunstan DW, Carson V, Salmon J. Mediating effects of dietary intake on associations of TV viewing, body mass index and metabolic syndrome in adolescents. Obes Sci \& Prac. 2016;2(3):232-40.

17. Garnett SP, Cowell CT, Baur LA, Fay RA, Lee J, Coakley J, Peat JK, Boulton TJ. Abdominal fat and birth size in healthy prepubertal children. Int J Obes Relat Metab Disord. 2001;25(11):1667-73.

18. Kuczmarski RJ, Ogden CL, Guo SS, et al. 2000 CDC growth charts for the United States: Methods and development. National Center for Health Statistics Vital Health Stat 11. 2002;11(246):1-190.

19. Cole TJ, Bellizzi MC, Flegal KM, Dietz WH. Establishing a standard definition for child overweight and obesity worldwide: international survey. BMJ. 2000;320(7244):1240

20. Hardy L, Baur L, Garnett S, Crawford D, Campbell K, Shrewsbury V, Cowell C, Salmon J. Family and home correlates of television viewing in 12-13 year old adolescents: The Nepean Study. Int J Behav Nutr Phys Act. 2006;3(1):1-9.

21. Willett WC, Howe GR, Kushi LH. Adjustment for total energy intake in epidemiologic studies. Am J Clin Nutr. 1997;65(4 Suppl):1220S-8S.

22. Chastin SF, Granat MH. Methods for objective measure, quantification and analysis of sedentary behaviour and inactivity. Gait \& Posture. 2010;31(1):82-6

23. Mattocks C, Ness A, Leary S, Tilling K, Blair SN, Shield J, Deere K, Saunders J, Kirkby J, Smith GD, et al. Use of accelerometers in a large field-based study of children: protocols, design issues, and effects on precision. J Phys Act Health. 2008;5(s1):S98-S111.

24. Australian Bureau of Statistics: National Nutrition Survey 1997: Nutrient intakes and physical measurements (Report No. 4805.0). Canberra, Australia: Australian Bureau of Statistics; 1998

25. Duke PM, Litt IF, Gross RT. Adolescents' self-assessment of sexual maturation. Pediatrics. 1988:66(6):918-20.

26. Trost SG, Pate RR, Sallis JF, Freedson PS, Taylor WC, Dowda M, Sirard J. Age and gender differences in objectively measured physical activity in youth. Med Sci Sports Exerc. 2002;34(2):350-5. 
27. Mackinnon DP. Introduction to statistical mediation analysis. New York: Taylot \& Francis Group; 2008.

28. Preacher KJ, Hayes AF. SPSS and SAS procedures for estimating indirect effects in simple mediation models. Behav Res Methods Instrum Comput. 2004;36(4):717-31.

29. Krahnstoever Davison K, Marshall SJ, Birch LL. Cross-sectional and longitudinal associations between TV viewing and girls' body mass index, overweight status, and percentage of body fat. J Pediatr. 2006;149(1):32-7.

30. Danner FW. A national longitudinal study of the association between hours of TV viewing and the trajectory of BMI growth among US children. J Pediatr Psychol. 2008;33(10):1100-7.

31. Australian Health Survey: Physical activity, 2011-12 cat no. 4364.0.55.004. Canberra: Commonwealth of Australia; 2013. http://www.abs.gov.au

32. Australian Health Survey: updated results, 2011-2012. Overweight and obesity. http://www.abs.gov.au/ausstats/abs@.nsf/Lookup/ 33C64022ABB5ECD5CA257B8200179437?opendocument

33. Carson V, Janssen I. Volume, patterns, and types of sedentary behavior and cardio-metabolic health in children and adolescents: a cross-sectional study. BMC Public Health. 2011;11:274.

34. Ekelund U, Luan J, Sherar LB, Esliger DW, Griew P, Cooper A. Moderate to vigorous physical activity and sedentary time and cardiometabolic risk factors in children and adolescents. JAMA. 2012;307(7):704-12.

35. Collings PJ, Wijndaele K, Corder K, Westgate K, Ridgway CL, Sharp SJ, Atkin AJ, Bamber D, Goodyer I, Brage $\mathrm{S}$, et al. Prospective associations between sedentary time, sleep duration and adiposity in adolescents. Sleep Med. 2015;16(6):717-22.

36. Barr-Anderson DJ, Larson NI, Nelson MC, Neumark-Sztainer D, Story M. Does television viewing predict dietary intake five years later in high school students and young adults? Int I Behav Nutr Phys Act. 2009;6:7.

37. Larson NI, Neumark-Sztainer DR, Story MT, Wall MM, Harnack LJ, Eisenberg ME. Fast food intake: longitudinal trends during the transition to young adulthood and correlates of intake. J Adolesc Health. 2008;43(1):79-86.

38. Kelly B, Halford JCG, Boyland EJ, Chapman K, Bautista-Castaño I, Berg C, Caroli M, Cook B, Coutinho JG, Effertz T, et al. Television food advertising to children: a global perspective. Am J Pub Health. 2010;100(9):1730-6.

39. Rosenheck R. Fast food consumption and increased caloric intake: a systematic review of a trajectory towards weight gain and obesity risk. Obes Rev. 2008;9(6):535-47.

40. Malik VS, Schulze MB, Hu FB. Intake of sugar-sweetened beverages and weight gain: a systematic review. Am J Clin Nutr. 2006;84(2):274-88.

41. Collins CE, Watson J, Burrows T. Measuring dietary intake in children and adolescents in the context of overweight and obesity. Int J Obes. 2010;34(7):1103-15.

42. Fuller-Tyszkiewicz M, Skouteris H, Hardy LL, Halse C. The associations between TV viewing, food intake, and BMI. A prospective analysis of data from the Longitudinal Study of Australian Children. Appetite. 2012;59(3):945-8.

\section{Submit your next manuscript to BioMed Central and we will help you at every step:}

- We accept pre-submission inquiries

- Our selector tool helps you to find the most relevant journal

- We provide round the clock customer support

- Convenient online submission

- Thorough peer review

- Inclusion in PubMed and all major indexing services

- Maximum visibility for your research

Submit your manuscript at www.biomedcentral.com/submit

) Biomed Central 